\title{
Les représentations sociales de la santé publique chez les professionnels de santé dans les armées françaises
}

\author{
Social representations of public health among healthcare workers within the French Armed Forces \\ M.A. Sanchez*, G. Velut, P. Nivoix, A. Mayet, L. Dany, J.B. Meynard, X. Deparis, F. Berger \\ Centre d'épidémiologie et de santé publique des Armées de Marseille, camp de Sainte-Marthe, 408, rue Jean-Queillau, 13014 Marseille, France \\ Reçu le 28 août 2014 ; accepté le 19 octobre 2015 \\ Disponible sur Internet le 31 décembre 2015
}

\begin{abstract}
Background. - Public health is a multidisciplinary activity whose fields of action are acquiring an increasingly broad. The Service de santé des armées (SSA) has always had a culture of public health problems thanks to doctors specialized in the treatment of major diseases. Often involved in public health activities, health professionals nevertheless have a fragmented vision. The objective was to describe the social representations of public health of military healthcare workers.

Method. - The responders were doctors, nurses, veterinarians and pharmacists practicing in different areas of SSA (caregivers, administrators, policy makers) and were interviewed by telephone. A question of spontaneous evocation on the representations of public health in the army was asked. The overall lexical analysis was performed according to the method of rank-frequency. Categorical analysis was conducted to better understand the whole lexical field use.

Results. - There were 90 responders. The most salient terms were "prevention, epidemiological surveillance and vaccination". The categorical analysis showed that doctors used a lexical field primarily focused on diseases and risk behaviors, nurses on the specifics of military surveillance and policy makers about the cross-discipline.

Conclusion. - Public health in the army is mainly represented by epidemiological surveillance and prevention. Given the non-mentioned fields, a strengthening of communication on the current challenges of public health would probably improve adherence of healthcare professionals since public health takes on more and more importance in the development of the national health policy and management of health crises.
\end{abstract}

(C) 2015 Elsevier Masson SAS. All rights reserved.

Keywords: Public health; Representation; Army; Healthcare worker

\section{Résumé}

Position du problème. - La santé publique est une activité multidisciplinaire dont les champs d'action sont de plus en plus nombreux. Le Service de santé des armées (SSA) a toujours eu une culture de la santé publique grâce notamment aux médecins des grandes endémies. Souvent impliqués dans des activités de santé publique les professionnels de santé en ont pourtant une vision parcellaire. L'objectif était de décrire les représentations sociales de la santé publique des professionnels de santé militaires.

Méthode. - Les répondants étaient des médecins, infirmiers, vétérinaires et pharmaciens exerçant dans les différents domaines du SSA (soignants, administratifs, décideurs) et ont été interrogés par téléphone. Une question d'évocations spontanées portant sur les représentations de la santé publique dans les armées leur a été posée. L'analyse lexicale globale a été effectuée selon la méthode des rang-fréquence. Une analyse catégorielle a été réalisée pour mieux appréhender l'ensemble du champ lexical utilisé.

Résultats. - Le nombre de répondants a été de 90. Les termes les plus saillants étaient « prévention, surveillance épidémiologique et vaccination ». L'analyse catégorielle a montré que les médecins des forces avaient un champ lexical principalement axé sur les maladies et les conduites à risque, les infirmiers sur les spécificités de la surveillance militaire et les décideurs sur la politique de santé et l'organisation des systèmes.

* Auteur correspondant. 44, rue Castagnary, 75015 Paris.

Adresse e-mail : marc-antoine.sanchez@intradef.gouv.fr, eomsanchez@hotmail.com, survepid.despsud@sante.defense.gouv.fr (M.A. Sanchez). 
Conclusion. - L'image de la santé publique dans les armées est représentée essentiellement par la surveillance et la prévention. Compte tenu des champs d'activités non évoqués, un renforcement de la communication sur les défis actuels et la valorisation des activités de la santé publique permettrait sans doute d'améliorer l'adhésion des acteurs à leur mission de santé publique, au moment où elle revêt une importance majeure dans le développement des politiques de santé et dans la gestion des crises sanitaires.

(C) 2015 Elsevier Masson SAS. Tous droits réservés.

Mots clés : Santé publique ; Représentation ; Armée ; Professionnel de santé

\section{Introduction}

Le Service de santé des armées (SSA) a toujours eu une culture de la santé publique grâce aux médecins qui ont contribué à des avancées majeures et novatrices dans ce domaine, notamment en médecine tropicale avec la lutte contre la trypanosomiase africaine [1] ou la prise en charge de la méningite en Afrique sub-saharienne [2], et récemment en anticipant les modifications du calendrier vaccinal national concernant la rougeole [3], en élaborant un système de surveillance en temps réel (dispositif d'Alerte et de Surveillance en Temps Réel [ASTER] [4-6] ou en développant un service de veille sanitaire de défense performant $[7,8]$. Les champs d'actions de la santé publique dans le milieu militaire concernent aussi bien l'épidémiologie de terrain, la veille sanitaire de défense, la surveillance épidémiologique, la prévention, la promotion de la santé, la recherche clinique, l'évaluation des pratiques professionnelles et les politiques de santé. À la suite d'une grande enquête menée par la Caisse nationale militaire de sécurité sociale et le SSA auprès des militaires et de leur famille, des plans d'actions spécifiques ont été mis en place, comme le plan « troubles psychiques post-traumatiques dans les forces armées », le plan « alcool» et le plan «traumatismes liés aux activités physiques et sportives » [9-11].

Les acteurs principaux impliqués dans ces actions de santé publique militaire sont :

- la direction centrale du Service de santé des armées, qui donne les orientations politiques, les priorités d'actions et organise l'offre de soin ;

- le Centre d'épidémiologie et de santé publique des armées (CESPA), organisme dédié à la promotion de la santé, à la surveillance épidémiologique, à l'investigation de terrain et à la recherche clinique ;

- les hôpitaux d'instruction des armées et les centres médicaux des Armées, composés de médecins et d'infirmiers en contact permanent avec la population militaire et la population civile dans les hôpitaux et qui assurent l'offre de soins.

Le projet d'évolution du SSA à l'horizon 2020 prévoit une franche implication dans le domaine de la santé publique [12], en développant la recherche et l'ouverture au service public. Dans ce cadre, le CESPA travaille en étroite collaboration avec les acteurs civils nationaux et les acteurs internationaux militaires [13]. Les professionnels de santé du SSA, quels que soient leur métier ou leurs compétences, sont impliqués dans les activités de santé publique, dans le cadre de la surveillance, des investigations épidémiologiques, de la recherche et des actions en santé. Il existe des spécificités à la pratique de la santé publique dans le milieu militaire : il s'agit en effet d'une collectivité, très mobile et soumise à de fortes contraintes en termes d'exposition à des événements sanitaires physiques ou psychiques, en particulier lors des opérations extérieures (OPEX). Les médecins et infirmiers des forces bénéficient par conséquent d'une formation initiale renforcée en épidémiologie et en santé publique.

Il est difficile aujourd'hui de définir la santé publique de façon consensuelle tant les champs d'action sont de plus en plus vastes [14]. Les enjeux actuels en termes de structuration de la santé publique ou en matière de développement sont nombreux $[15,16]$. La santé publique peut s'apparenter à « la prise en charge collective de la santé d'une population dans son milieu de vie, qu'il s'agisse de soins, de prévention, d'éducation ou d'hygiène sociale » comme cela a été défini par l'Organisation mondiale de la santé. Les représentations de la santé publique par les professionnels de santé ont été peu étudiées. Une étude réalisée parmi des infirmiers hospitaliers montrait une vision de la santé publique réduite aux activités éducatives [17]. La participation active à une politique de santé était peu envisagée, restreignant la pratique à une relation soignant-patient très individuelle et non collective ou bien à des actions environnementales [17]. La majorité des personnels interrogés ne semblaient pas envisager de formations spécifiques, alors que par exemple se développe actuellement en milieu hospitalier le champ de l'éducation thérapeutique du patient, qui demande des compétences particulières [17]. La place de la santé publique et sa perception par les autres acteurs de santé a toujours été ambivalente, les activités des médecins de santé publique étant le plus souvent mal connues par leurs confrères $[18,19]$, ce qui peut sembler paradoxal au moment où cette spécialité prend de l'importance, tant dans le secteur public que dans le secteur privé de l'industrie pharmaceutique ou du conseil [18,20-22]. Il en est de même au sein du SSA où le CESPA tend aujourd'hui à se développer pour mieux répondre à ses objectifs. La transversalité de la santé publique, interagissant avec le monde médical, scientifique, économique et politique la rend toujours aussi difficile à définir aujourd'hui qu'il y a vingt ans tant son domaine d'action est large [23]. Néanmoins, la culture et les méthodes de la santé publique ont progressé depuis quelques années, marquées par la loi de santé publique en 2004 [23] et la mise en place de nouvelles structures (agence régionale de santé, inscription des Observatoires régionaux de santé à la loi Hôpitaux, patients, santé et territoires en 2009). 
L'image de la santé publique qu'ont les professionnels de santé a été peu étudiée en général et n'a jamais fait l'objet de recherches dans les armées. Tous les professionnels de santé des armées sont impliqués dans des activités de santé publique. Une vision parcellaire, incomplète ou négative de la santé publique pourrait limiter la conscience d'un tel rôle dans leurs actions. À l'heure de la réorganisation du SSA, il semblait nécessaire de décrire les représentations sociales de la santé publique dans les armées. Une représentation sociale est définie comme "un ensemble organisé d'informations, d'opinions, d'attitudes et de croyances à propos d'un objet donné » qui possède un contenu et une organisation [24]. Cette approche des représentations permet une description plus globale que la simple représentation de l'image d'un objet, car elle permet une approche structurale des représentations grâce à la théorie dite du noyau central, et vise à décrire comment et pourquoi les représentations sont ainsi faites $[24,25]$.

L'objectif de cette étude était de décrire les représentations sociales de la santé publique qu'ont les professionnels de santé des armées, afin de mieux comprendre l'image actuelle de la santé publique dans ce contexte, et d'envisager des axes de développement susceptibles d'améliorer l'implication des différents acteurs.

\section{Population et méthode}

\subsection{Population et recueil de données}

Il s'agissait d'une étude descriptive transversale réalisée par téléphone. La population cible était celle des militaires d'active du SSA (médecins, pharmaciens, vétérinaires, infirmiers) joignables par téléphone du $1^{\mathrm{er}}$ au 30 avril 2014 pendant les heures ouvrables. La base de sondage était issue des annuaires officiels et actualisés des organismes sélectionnés. L'échantillonnage a été réalisé par un sondage aléatoire à deux degrés par type d'organisme, en métropole (Centres médicaux des armées, Force d'action navale, directions régionales du Service de santé des armées [DRSSA], direction centrale du Service de santé des armées [DCSSA], hôpitaux d'instruction des armées [HIA], Écoles, Institut de recherche biologique des armées), en outremer (direction inter-armées du Service de santé [DIASS]) et en opération extérieure (OPEX) (Commandement santé en OPEX), ainsi que par type de personnels (médecins des forces, infirmiers des forces, vétérinaires des armées, pharmaciens des armées, médecins spécialistes, médecins décideurs). Les médecins décideurs étaient des médecins appartenant à la DCSSA, aux HIA, DRSSA, DIASS, Écoles ou présents en OPEX et occupant des postes de direction, comme les directeurs d'hôpitaux par exemple, ce qui constitue une spécificité du SSA.

Pour chacun des organismes sélectionnés aléatoirement et par type de personnel, le premier personnel du SSA disponible a été interrogé. Si aucun personnel ne pouvait être interrogé la structure suivante était sélectionnée, jusqu'à obtention de l'objectif fixé par strate. L'effectif fixé a priori était de 100 enquêtés, répartis de la manière suivante : 31 médecins décideurs, 20 médecins des forces, 20 infirmiers des forces,
17 médecins hospitaliers, 6 vétérinaires, 6 pharmaciens. En raison du faible effectif du groupe des personnels « décideurs », des relances ont été effectuées jusqu'à obtention de l'effectif fixé. Les décideurs, qui jouent un rôle important sur la politique de santé dans les armées, ont été surreprésentés afin d'affiner la compréhension de leurs représentations de la santé publique.

\subsection{Protocole}

Le recueil de données a eu lieu entre le 15 avril et le 31 mai 2014. Ces dates ont été modifiées par rapport aux dates fixées initialement pour interroger le maximum de professionnels et répondre au mieux aux objectifs quantitatifs. Les personnels ont été contactés par téléphone sur leur lieu de travail pendant les heures ouvrables en prenant en compte l'éventuel décalage horaire selon le territoire. L'intérêt du recueil de données par téléphone était d'obtenir des réponses spontanées en limitant le délai de réflexion et de pouvoir interroger les personnels peu accessibles par Internet ou par courrier (OPEX, bâtiments de la Marine nationale).

Pour rendre l'interrogatoire le plus neutre possible, l'enquêteur qui était médecin a été formé pendant une journée et n'était pas rattaché au CESPA. Avant de collecter les données, l'enquêteur se présentait et décrivait l'étude de façon standardisée. Le questionnaire était volontairement court et rapide, estimé à environ 3 minutes, afin de limiter l'interruption possible de la conversation et le risque de données manquantes. Ce questionnaire téléphonique a été testé parmi 10 volontaires pour valider son contenu et la méthodologie.

Une autorisation a été demandée à la DCSSA pour réaliser l'étude. Un avis consultatif a été demandé auprès du Comité de protection des personnes Sud Méditerranée I.

\subsection{Mesures}

L'âge, le grade et l'ancienneté ont été recueillis au début de l'entretien téléphonique ; les sujets devaient ensuite répondre à la question suivante : "Pourriez-vous donner 5 mots ou expressions qui vous viennent à l'esprit quand on vous évoque la santé publique dans les armées, en les classant du plus important au moins important?».

Le logiciel Epi Info 7 (CDC Atlanta) a été utilisé pour la saisie. L'analyse statistique a été réalisée avec le logiciel Stata 11 (Stata Corporation). L'analyse lexicale a été effectuée avec le logiciel EVOC 2000 permettant la mise en évidence du contenu des représentations à partir du croisement de la fréquence d'apparition des termes et de leur rang moyen selon la méthode des rang-fréquence [24]. Cette méthode, appelée aussi analyse prototypique, permet de mettre en évidence la structure des représentations sociales, qui sont un ensemble organisé d'informations, d'opinions, de croyances [24,26]. La théorie du noyau central repose sur l'hypothèse qu'une représentation sociale comprend un « noyau central » et un « système périphérique » [25,27]. L'intérêt de cette méthode est de donner de l'importance à des groupes de mots plutôt qu'à d'autres et de pouvoir ainsi interpréter leurs relations et l'organisation des représentations. Selon M.L. Rouquette, « une 
représentation se définit par 2 composantes : ses éléments constitutifs d'une part, et son organisation », c'est-à-dire les relations qu'entretiennent ces éléments [28]. Le croisement de la fréquence et du rang des termes permet d'obtenir un tableau, dit tableau de Vergès, comprenant 4 cases : la zone centrale (noyau central), rassemblant les éléments forts des représentations, c'est-à-dire les mots les plus « importants » et les plus fréquemment cités, les 2 zones potentielles de changement, et la périphérie extrême, qui composent le système périphérique. La périphérie extrême regroupe les éléments faibles des représentations, rarement cités et en dernière position [25]. Le système central est lié à la mémoire collective et à l'histoire du groupe. Il est consensuel et définit l'homogénéité du groupe. Le système périphérique permet l'intégration des expériences individuelles. Les zones potentielles de changement dans lesquelles se trouvent les mots ayant une fréquence de citation élevée sont des zones ambiguës dans lesquelles peuvent se trouver des éléments de changements des représentations, c'est-à-dire des éléments permettant l'adaptation des représentations aux évolutions du contexte. D'après J.C. Abric le système périphérique constitue " l'interface entre le noyau central et la situation concrète dans laquelle s'élabore la représentation » [24]. Pour la construction du carré de Vergès, le rang moyen a été fixé à 2,5 et la fréquence supérieure ou égale à 10 . Ces critères ont été choisis en fonction du nombre d'évocations demandées aux répondants, et de la fréquence des termes les plus cités.

Une analyse catégorielle a été réalisée à partir de l'ensemble du champ sémantique utilisé par trois catégories de répondants nous intéressant particulièrement : les catégories les plus fréquentes (médecins et infirmiers des forces), et la catégorie la plus influente (décideurs). Le choix des groupes sémantiques a été réalisé a posteriori par 2 enquêteurs afin de pouvoir interpréter l'ensemble des termes évoqués. Le regroupement sémantique a été réalisé par association afin de dégager des thématiques communes. À partir des résultats, 5 groupes sémantiques ont été créés pour faciliter l'interprétation selon les variables utilisées dans le questionnaire. L'intérêt de distinguer les représentations des différentes catégories de répondants était de connaître l'image de la santé publique de professionnels de santé ayant des pratiques différentes. Le choix des groupes sémantiques a été réalisé de la façon la plus cohérente possible selon l'investigateur pour en faciliter l'interprétation. Chaque terme a été classé dans l'un des groupes suivants : mot en rapport avec une maladie, mot se rapportant à la santé publique en tant que discipline scientifique, mot spécifique à la santé publique dans les armées, mot à connotation négative et mot à connotation positive vis-à-vis de la spécialité.

\section{Résultats}

\subsection{Description de la population d'étude}

Les caractéristiques des répondants sont décrites dans le Tableau 1. Six personnels n'ont pas pu être contactés malgré des relances itératives. Quatre-vingt-dix ont répondu au questionnaire et 4 ont refusé de participer. Les motifs de refus étaient : le manque de temps $(n=2)$, le désintérêt pour le sujet $(n=1)$ et le manque de confidentialité des réponses par téléphone $(n=1)$. Les hommes représentaient 76,6\% des répondants. Le grade le plus représenté était celui de médecin en chef (46,2\%). L'âge moyen des répondants était de 45 ans avec une ancienneté moyenne de 24 ans de service. Les médecins décideurs ont été moins nombreux à répondre que prévu $(n=25)$.

Tableau 1

Répartition par structure et par fonction des effectifs réalisés et attendus pour les personnels de santé du Service de santé des armées interrogés par téléphone entre le 15 avril et le 31 mai 2014.

\begin{tabular}{|c|c|c|c|c|c|c|c|}
\hline & $\begin{array}{l}\text { Décideurs } \\
\text { Réa. (at.) }\end{array}$ & $\begin{array}{l}\text { Médecins } \\
\text { des forces } \\
\text { Réa. (at.) }\end{array}$ & $\begin{array}{l}\text { Infirmiers } \\
\text { des forces } \\
\text { Réa. (at.) }{ }^{\text {a }}\end{array}$ & $\begin{array}{l}\text { Vétérinaires } \\
\text { Réa. (at.) }{ }^{\mathrm{a}}\end{array}$ & $\begin{array}{l}\text { Pharmaciens } \\
\text { Réa. (at.) }\end{array}$ & $\begin{array}{l}\text { Autres médecins } \\
\text { Réa. (at.) })^{\mathrm{a}}\end{array}$ & $\begin{array}{l}\text { Totaux } \\
\text { Réa. (at.) }\end{array}$ \\
\hline \multicolumn{8}{|l|}{ Marine } \\
\hline Centres médicaux & & $3(3)$ & $3(3)$ & & & & $6(6)$ \\
\hline \multicolumn{8}{|l|}{ Outre-mer } \\
\hline Direction & $3(3)$ & & & $1(2)$ & $0(2)$ & & $4(7)$ \\
\hline Centres médicaux & & $4(3)$ & $4(3)$ & & & & $8(6)$ \\
\hline \multicolumn{8}{|l|}{ Opération extérieure } \\
\hline État-major santé & $2(4)$ & & & $1(2)$ & & & $3(6)$ \\
\hline Centres médicaux & & $5(4)$ & $5(4)$ & & & & $10(8)$ \\
\hline Centres médicaux & & $10(10)$ & $10(10)$ & & & & $20(20)$ \\
\hline Direction régionale & $3(3)$ & & & $2(2)$ & $2(2)$ & $3(3)$ & $10(10)$ \\
\hline \multicolumn{8}{|l|}{ Métropole } \\
\hline Hôpitaux & $4(4)$ & & & & $2(2)$ & $10(10)$ & $16(16)$ \\
\hline Écoles & $4(4)$ & & & & & & $4(5)$ \\
\hline Institut de recherche biomédicale & $0(1)$ & & & & & $4(4)$ & $4(5)$ \\
\hline Direction centrale & $9(12)$ & & & & & & $9(12)$ \\
\hline \multicolumn{8}{|l|}{ Totaux } \\
\hline & $25(31)$ & $22(20)$ & $22(20)$ & $4(6)$ & $4(6)$ & $17(17)$ & $94(100)$ \\
\hline
\end{tabular}

\footnotetext{
${ }^{\text {a }}$ Réa. (at.) : effectifs réalisés (effectifs attendus).
} 
Tableau 2

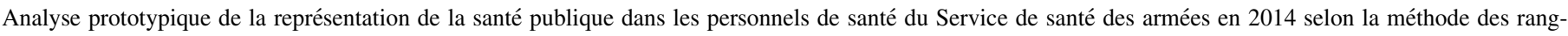
fréquence.

\begin{tabular}{|c|c|c|c|c|c|c|}
\hline \multirow[b]{4}{*}{ Fréquence } & \multicolumn{6}{|l|}{ Rang moyen d'apparition } \\
\hline & \multicolumn{3}{|l|}{$\leq 2,5$} & \multicolumn{3}{|l|}{$>2,5$} \\
\hline & \multicolumn{3}{|l|}{ Zone centrale } & \multicolumn{3}{|l|}{ Zone potentielle de changement } \\
\hline & Mot & Fréquence $(n)$ & Rang & Mot & Fréquence $(n)$ & Rang \\
\hline \multirow{5}{*}{$\geq 10$} & Prévention & 45 & 2,5 & & & \\
\hline & Surveillance épidémiologique & 30 & 2,2 & Opération extérieure & 11 & 2,9 \\
\hline & $\begin{array}{l}\text { Message épidémiologique } \\
\text { hebdomadaire }\end{array}$ & 10 & 2,5 & & & \\
\hline & Vaccination & 29 & 2,5 & & & \\
\hline & \multicolumn{3}{|l|}{ Zone potentielle de changement } & \multicolumn{3}{|l|}{ Périphérie } \\
\hline \multirow[t]{9}{*}{$<10$} & $\begin{array}{l}\text { Centre d'épidémiologie et de } \\
\text { santé publique des armées }\end{array}$ & 7 & 1,6 & Statistique & 8 & 2,8 \\
\hline & Contraignant & $4^{\mathrm{a}}$ & 1,8 & Compétence & 7 & 3,3 \\
\hline & & & & Education & 7 & 3,2 \\
\hline & & & & Enquête & 7 & 3,5 \\
\hline & & & & Hygiène & 7 & 3,3 \\
\hline & & & & Médecine collectivité & 5 & 3 \\
\hline & & & & Paludisme & 5 & 2,6 \\
\hline & & & & Visite médicale périodique & 5 & 3,4 \\
\hline & & & & Manque de rétro-information & $4^{\mathrm{a}}$ & 3,3 \\
\hline
\end{tabular}

a La fréquence minimale des termes dans le tableau était de 4.

\subsection{Représentations sociales de la santé publique dans les armées}

Les résultats sont présentés dans le Tableau 2. À la question posée, 190 termes différents ont été cités. Les répondants ont donné de 0 à 5 mots avec une moyenne de 4,3 mots sur les 5 demandés. Les termes les plus fréquents et les mieux placés (fréquence supérieure à 10, rang inférieur ou égal à 2,5) étaient prévention, surveillance épidémiologique, message épidémiologique hebdomadaire et vaccination, et formaient le noyau central. Le terme de la première zone potentielle de changement, avec une fréquence de citation supérieure à 10 et un rang supérieur à 2,5 (moins « importante »), était opération extérieure. Les termes CESPA et contraignant étaient peu fréquemment cités (fréquence de citation inférieure à 10) mais de plus grande importance (rang inférieur à 2,5), ils représentaient la deuxième zone de changement. La zone périphérique (fréquence de citation inférieure à 10, rang supérieur à 2,5) comprenait les termes statistiques, compétence, éducation, enquête, hygiène, paludisme.

\subsection{Analyse catégorielle}

Les résultats sont présentés dans le Tableau 3. Indépendamment des catégories d'individus interrogés, la plupart des termes évoqués concernaient la santé publique en tant que spécialité médicale. Les médecins des forces évoquaient principalement des termes en rapport avec les maladies tropicales, les conduites à risque ou les maladies chroniques, préoccupations actuelles de santé publique. La santé publique dans les armées leur paraissait être une contrainte dans leurs activités quotidiennes et les données transmises pour la surveillance ne leur semblaient pas toujours être exploitées à bon escient. Le CESPA était cité par tous les groupes comme étant l'acteur principal de santé publique du SSA. Les termes à connotation positive étaient moins présents mais la compétence, la disponibilité, la nécessité d'un service de santé publique performant étaient des termes exprimés par tous les groupes. Les infirmiers avaient un vocabulaire riche concernant les spécificités militaires de la discipline, et les termes opération extérieure, déclaration, visite médicale périodique étaient saillants. Ils évoquaient un manque de formation en santé publique et pensaient que la santé publique et les différentes activités qu'elle représente n'étaient pas assez développées. Les médecins décideurs avaient un vocabulaire riche concernant les différents aspects que peut prendre la santé publique actuelle, les termes vaccination, éducation, enquête, coopération, démarche qualité, parcours de soin et parcours de santé étaient cités comme des activités de santé publique. Ils évoquaient également certains acteurs civils comme le Département d'information médicale et l'agence régionale de santé.

Il n'y avait pas de différence notable de répartition des termes en fonction du contexte d'emploi (métropole, outre-mer et OPEX).

\section{Discussion}

\subsection{Forces et faiblesses}

L'analyse prototypique présente l'intérêt majeur de ne pas chercher à évaluer les connaissances des répondants mais d'accéder à leur univers représentationnel et à la structure des représentations sociales. Cette méthode originale nous a permis d'obtenir des résultats informatifs en fonction des catégories de 
Tableau 3

Analyse catégorielle de l'image de la santé publique en fonction de l'emploi des personnels de santé du Service de santé des armées en 2014.

\begin{tabular}{|c|c|}
\hline \multirow[t]{2}{*}{ Thème } & Fonction \\
\hline & Médecin d'unité \\
\hline $\begin{array}{l}\text { Maladie et conduites } \\
\text { à risque }\end{array}$ & $\begin{array}{l}\text { Paludisme (3), infection sexuellement } \\
\text { transmissible (3), exposition sexuelle à risque } \\
\text { (2), chikungunya (2), maladie déclaration } \\
\text { obligatoire (2), SIDA, addiction, alcoolisme, } \\
\text { cannabis, dépistage, effet secondaire, } \\
\text { leishmaniose, maladie tropicale, syndrôme de } \\
\text { stress post-traumatique, toxi-infection } \\
\text { alimentaire collective, traumatisme sonore } \\
\text { aigu, surpoids }\end{array}$ \\
\hline $\begin{array}{l}\text { Spécialité santé } \\
\text { publique }\end{array}$ & $\begin{array}{l}\text { Surveillance épidémiologique }{ }^{\mathrm{a}}(13), \\
\text { prévention (13), vaccination (9), médecine des } \\
\text { collectivités (3), campagne d'information (2), } \\
\text { conseil (2), éducation (2), statistique (2), } \\
\text { analyse, base de données, conduite à tenir, } \\
\text { communication, enquête, humanité, } \\
\text { impartialité, Institut national de veille sanitaire, } \\
\text { recherche clinique, surveillance, soutien } \\
\text { méthodologique }\end{array}$ \\
\hline
\end{tabular}

Prévention (13), vaccination (5), surveillance épidémiologique ${ }^{\mathrm{a}}(4)$, hygiène $(2)$, médecine collectivité (2), statistique (2), administratif, expertise, garantie des soins, humanitaire, médecine différente, procédure, prophylaxie, social, soutien méthodologique

Décideur

Dengue, lutte anti-vectorielle, obésité, tabagisme, alcoolisme, paludisme

Prévention (15), surveillance épidémiologique ${ }^{\mathrm{a}}(9)$, vaccination (8), éducation (4), coopération (2), prophylaxie (2), enquête (2), action, agence régionale de santé, collectivité, communautaire, déclaration, démarche qualité, département d'information médicale, endémie, hygiène, intérêt général, parcours de santé, parcours de soin, politique, priorité, projet d'établissement, recueil de données, réseau, scientifique, statistique

\section{Spécificité militaire

\begin{abstract}
Message épidémiologique hebdomadaire (7), CESPA (4), fiche spécifique de déclaration ${ }^{\mathrm{b}}$ (3), aptitude, compte rendu, médecine d'unité, rapport commandement, survepid ${ }^{\mathrm{c}}$, bulletin épidémiologique des armées, opération
\end{abstract} extérieure}

Image négative

Image positive
Contraignant (2), doctrine changeante, effectif insuffisant, manque de centralisation, manque de crédibilité, manque de moyen, manque de formation, travaillent pour eux
Message épidémiologique hebdomadaire (3), opération extérieure (2), déclaration (2), Département d'épidémiologie et de santé publique, information, moins présent que dans le civil, référent, soutien sanitaire, soutien activité sportive, visite médicale périodique ${ }^{\mathrm{d}}$, bulletin épidémiologique des armées

Manque de rétro-information (3), manque de formation, pas actualisé, pas assez développé, relation hôpital praticien, contraignant
Opération extérieure (4), visite médicale périodique $^{\mathrm{d}}(3)$, maintien en condition opérationnelle (2), Marseille (2), CESPA (2), médecine d'armée, message d'alerte, Océan indien

Inutile, isolé, pauvre, contraignant

Nécessité, obligatoire, reconnu, sérieux, utile, compétence, disponibilité

\footnotetext{
${ }^{a}$ Surveillance systématique et continue d'événements de santé définis.

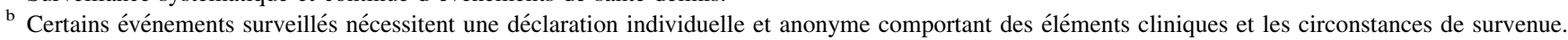

${ }^{c}$ Surveillance épidémiologique.

${ }^{\mathrm{d}}$ Visite médicale obligatoire pour tous les militaires dans le cadre de la médecine de prévention.
}

personnels interrogés. L'analyse catégorielle construite en appui de l'analyse prototypique fait intervenir une part de subjectivité dans le regroupement sémantique des termes. Cependant, les thèmes ont été choisis en tenant compte des résultats obtenus à la suite de l'analyse prototypique.

Les délais de réalisation ont été plus longs que ceux attendus en raison de la difficulté du recueil des données. Les répondants devaient être joints par téléphone durant leurs horaires de travail et être disponibles. Les répondants présents en outre-mer étaient difficilement joignables compte tenu du décalage horaire, les répondants en OPEX peu disponibles compte tenu des contraintes opérationnelles. Les décideurs ont été les plus difficiles à interroger. Finalement jusqu'à 3 relances ont été faites, puis un courriel leur a été envoyé pour convenir d'un rendez-vous téléphonique. Ces efforts ont permis d'obtenir un taux de réponse de $90 \%$. La répartition selon le type d'organisme était très semblable à celle prévue par le protocole. Seulement 4 personnes n'ont pas souhaité répondre et nous ne pouvons pas déduire a priori l'intérêt qu'elles portent ou non à la santé publique.

L'investigateur était lui-même professionnel de santé du SSA et non rattaché au CESPA. Les répondants pouvaient être tentés d'employer un vocabulaire moins négatif ou péjoratif puisqu'ils étaient directement interrogés par l'auteur de l'étude. Cependant l'objectif était de garantir la spontanéité des réponses recueillies grâce au court délai de réflexion qui était imposé.

La majorité des médecins des forces interrogés étaient des médecins en chef, qui ont plus d'expérience et de responsabilités que les médecins commençant leur carrière. La plupart d'entre eux n'avait pas eu la même formation en santé publique que leurs confrères plus jeunes, ce qui a pu influer sur la construction de leurs représentations. 
Cette étude originale nous a néanmoins permis d'explorer l'univers représentationnel de la santé publique au sein du SSA, ce qui constitue à nos yeux un intérêt particulier à l'heure où elle tend à jouer un rôle incontournable dans la prise de décision, que ce soit sur le terrain par les médecins et infirmiers des forces ou dans les institutions de commandement par les médecins et cadres décideurs.

La pratique de la santé publique dans les armées ne peut être comparée à celle du système de santé civil français dans son ensemble, car ses objectifs et son fonctionnement sont différents. Cependant, les principales activités de santé publique sont représentées dans les armées. De plus, la formation en santé publique des professionnels de santé militaires est différente de celle des professionnels civils : outre la formation universitaire, ils suivent des formations spécifiques dispensées par l'institution. Pour ces raisons, nos résultats ne peuvent être généralisés, mais constituent des pistes des réflexions en vue d'autres études qui pourraient être menées auprès des acteurs de santé civils.

\subsection{Analyse des résultats}

Les principaux résultats de l'étude du noyau central ont montré une représentation forte de la santé publique dans les domaines de la prévention et de la surveillance. Ces termes étaient en lien avec les principaux champs de la santé publique en général pour la prévention, et dans les armées pour la surveillance. La surveillance était une représentation forte, plus parce qu'elle fait partie intégrante des tâches imposées aux personnels de santé que parce qu'elle est perçue comme vraiment nécessaire. L'activité de prévention était clairement identifiée car les médecins et infirmiers des forces exercent quotidiennement des activités de prévention lors des visites médicales périodiques, qui peuvent s'assimiler à des visites de médecine du travail, lors des visites de mise en condition opérationnelle réalisées avant les missions, ou lors des séances de vaccination. Le terme opération extérieure, présent dans la zone potentielle de changement (fréquence supérieure à 10, rang supérieur à 2,5) était en lien avec les mesures préventives nécessaires à la préparation des militaires avant leur départ en mission, ainsi qu' au côté opérationnel perçu de l'épidémiologie de terrain. Les termes CESPA et contraignant, peu fréquemment cités, représentaient la deuxième zone de changement (fréquence inférieure à 10, rang inférieur à 2,5). Le terme CESPA était associé à la santé publique et le terme contrainte se rapportait au caractère obligatoire de la participation à la surveillance épidémiologique.

Notre étude montre que les professionnels de santé dans les armées ont des représentations partielles des champs de la santé publique. Les termes présents dans les zones de changements donnaient différentes informations : d'une part le terme opération extérieure évoquant l'épidémiologie de terrain contrastait avec la vision du noyau, centrée sur la prévention. Une autre vision moins administrative et plus opérationnelle, envisagée dans cette zone de contraste, montrait une évolution possible des représentations actuelles. D'autre part le CESPA, acteur principal de la santé publique dans les armées, était identifié comme susceptible de jouer un rôle dans le changement de ces représentations. Le terme contrainte était intéressant puisqu'il suggère que des termes à connotations négatives peuvent également apparaître selon ces changements. Cela est sans doute lié à une vision actuelle de la santé publique parfois perçue comme une tâche imposée. La zone périphérique comprenait des domaines d'actions de la santé publique dans lesquels les acteurs ont des compétences particulières, comme par exemple l'utilisation d'outils statistiques et la réalisation d'enquêtes. Là encore, la confusion existait avec la médecine des collectivités, notamment par l'activité des visites médicales périodiques. Il est intéressant de constater dans la périphérie un manque de rétro-information ressenti par les répondants concernant les actions de santé publique menées au sein du SSA. Il peut paraître surprenant que le terme recherche clinique ait été très peu cité, alors que celle-ci tend à se dynamiser et prend de plus en plus d'importance en France ainsi que dans le SSA [12,29].

Nos résultats ont montré le caractère non figé des représentations sociales de la santé publique dans les armées, leur caractère partiel et leurs contours flous. Les axes de changement des représentations, aujourd'hui tournées vers une vision administrative parfois contraignante des pratiques, qui ont été identifiés, pourraient s'envisager vers une vision plus opérationnelle et dynamique de la santé publique et cela grâce à son acteur principal, le CESPA. Cela permettrait peut-être une implication plus active des acteurs de santé et une meilleure connaissance de la santé publique et de son importance, mais cela reste à démontrer. La communication des acteurs de santé publique sur leurs activités et la promotion des champs d'application variés de la santé publique pourraient être des moyens d'actions.

L'analyse catégorielle a permis d'exploiter la richesse du lexique employé par les différents groupes de répondants ainsi que la diversité des champs lexicaux entre les groupes. Les infirmiers avaient une vision concrète et pratique de la santé publique, les médecins des forces une vision clinique par maladies, et les médecins décideurs une vision davantage politique et organisationnelle. Certains axes d'amélioration de la perception de la santé publique pourraient être suggérés à partir de cette analyse : les médecins et infirmiers des forces pourraient être sensibilisés aux différents champs d'actions de la santé publique et plus impliqués dans les actions à mener, notamment par la participation volontaire et active à la prévention, aux investigations, à la recherche ou même à la veille sanitaire. Les médecins décideurs, qui avaient une vision plus globale des champs d'action de la santé publique, pourraient être plus informés des activités de santé publique et des travaux réalisés en partenariat avec les autres institutions civiles ou militaires.

De même que dans l'étude réalisée auprès d'infirmiers hospitaliers [17], l'amélioration de la qualité de vie, le rôle du patient sur sa santé, la place des déterminants sociaux et environnementaux, l'approche globale et la transversalité des champs d'activités multidisciplinaires que représente la santé publique y étaient peu développées. Dans cette étude, la dimension individuelle de la relation patient-soignant était prépondérante, d'une part à cause de l'ancrage des représentations 
sociales des professionnels de santé interrogés dans leur pratique, mais également à cause d'une difficulté à se situer de façon plus globale dans un système et à en connaître les différentes missions [17].

\section{Conclusion}

Cette étude des représentations de la santé publique par les personnels médicaux et paramédicaux du Service de santé des armées était une étape préalable à l'évaluation de leurs besoins et attentes. En effet, perçus comme nécessaires, les différents aspects de la santé publique sont partiellement connus. Les professionnels de santé ont pour la plupart une vision de la santé publique centrée sur les spécificités militaires de la pratique de la santé publique. Les activités de santé publique princeps, notamment l'épidémiologie et la prévention, sont connues et bien identifiées. Toutefois la santé publique militaire reste un champ de la médecine collective plus secondaire qu'indispensable, présentant certaines difficultés dans sa mise en œuvre et pouvant parfois s'assimiler à une contrainte. Elle est de plus perçue comme un domaine d'experts mis en œuvre par des spécialistes, alors que les principaux acteurs de sa mise en œuvre sont les personnels de santé décideurs, les médecins et infirmiers des forces. En plus de donner une image de la santé publique qu'ont les professionnels de santé, ce travail permet d'envisager les pistes de réflexion et les leviers à mettre en place pour une participation plus forte des différents acteurs. Il sera complété par une étude quantitative en vue d'affiner l'image de la santé publique et de mieux comprendre les attentes des personnels du SSA.

\section{Déclaration de liens d'intérêts}

Les auteurs déclarent ne pas avoir de liens d'intérêts.

\section{Remerciements}

Nous remercions vivement le département de psychologie sociale et du travail de l'université Aix Marseille et plus particulièrement le professeur Themis Apostolidis sans qui ce travail n'aurait pas été possible.

Nous remercions Madame Liliane Pellegrin pour sa collaboration.

Nous remercions l'ensemble des personnels médicaux et paramédicaux qui ont participé à l'étude.

\section{Références}

[1] Milleliri JM. Jamot vu par Bado. Med Sante Trop 2012;22:22-8.

[2] Lapeyssonnie L. La méningite cérébro-spinale en Afrique. Genève: Organisation mondiale de la santé; 1963.

[3] Génicon C, Meynard JB, Duron S, Haus-Cheymol R, Ollivier L, Le Goff C, et al. Feedback on the management of the 2011 measles outbreak by French military general practitioners: an evaluation study. Rev Epidemiol Sante Publique 2014;62:119-26.

[4] Meynard JB, Chaudet H, Texier G, Queyriaux B, Deparis X, Boutin JP. Surveillance épidémiologique en temps réel dans les armées : concepts, réalités et perspectives en France. Rev Epidemiol Sante Publique 2008;56:11-20.
[5] Meynard JB, Chaudet H, Green A, Texier G, Webber D, et al. Proposal of a framework for evaluating military surveillance systems for early detection of outbreaks on duty areas. BMC Public Health 2008;8:146.

[6] Meynard JB, Chaudet H, Texier G, Dupuy B, Queyriaux B, Pellegrin. et al. Advantages and limits of real time epidemiological surveillance during military deployments: the experience of the French Armed Forces. Mil Med 2009;174:1068-74.

[7] Tanti M, Hupin C, Boutin JP, Hassanaly P. Un modèle de grille d'analyse des documents scientifiques : application à la veille sanitaire de défense. Documentaliste-Sciences de 1'Information 2010;1:4-12.

[8] Tanti M, Hupin C, Hassanaly P, Boutin JP. Processus de veille documentaire scientifique au profit de la veille sanitaire de défense. Humanisme et Entreprise 2007;5:65-86.

[9] Direction centrale du Service de santé des armées. Plan d'action « troubles psychiques post-traumatiques dans les forces armées » 2013-2015. «Lutte contre le stress opérationnel et le stress post-traumatique »; 2013 [consulté le 10.07.2014 sur 1'URL : http://www.defense.gouv.fr/layout/set/print/ content/download/233438/2610358/file/PLAN-Action-ESPT-Web.pdf].

[10] De Montleau F. Besoins de prévention des militaires d'active. Les risques liés à l'alcool en milieu militaire. Med Armees 2010;38:37-48.

[11] Bigard X, Cravic JY, Banzet S. Prévention des risques liés à la préparation physique du militaire : synthèse des connaissances actuelles. Med Armees 2010;38:07-16.

[12] Direction centrale du Service de santé des armées. Le projet de service SSA 2020. Ministère de la Défense et des Anciens combattants; 2013 [Report no.: 515696].

[13] Denux V. L'interaction civilo-militaire médicale dans la résolution des crises. Med Armees 2013;41:231-4.

[14] Bourdillon F, Brücker G, Tabuteau D, editors. Traité de santé publique. 2nd ed. Médecine-Sciences Flammarion; 2007.

[15] Koplan JP, Fleming DW. Current and future public health challenges. JAMA 2000;284:1696-8.

[16] Alla F, Borgès Da Silva G. Pour une culture partagée de la promotion de la santé et des soins. Sante Publique 2013;25:5.

[17] Pommier J, Laurent-Beq A, Beurrier B, Fidan S, Guilhem L, Jeandel L, et al. La perception de la santé publique des infirmières à l'hôpital. Sante Publique 2004;16:383-92.

[18] Demeulemeester R, et al. HCSP. Une culture de santé publique en voie de développement : réflexions sur l'avenir de la formation des médecins en santé publique. Actualité et dossier en santé publique 1992;1:6-9.

[19] Fassin D. Comment faire de la santé publique avec des mots. Une rhétorique à l'œuvre. Ruptures revue transdisciplinaires en santé 2000;7:58-78.

[20] Alves de Rezende B. La formation des internes de santé publique en France. Offre et satisfaction. Bordeaux: Université Bordeaux 2-Victor Segalen; 2010 [Thèse d'exercice : médecine].

[21] Bricoteau D, Hoden S. Une nouvelle spécialité médicale : la santé publique, pour quoi faire ? Débouchés pour les internes de santé publique en France en 1990. Prospective.. Lille: Université Lille 2; 1990 [Thèse d'exercice : médecine].

[22] Heintzmann F, Vidal A, Chabot J, Brodin M, Lombrail P, Gottot S. Interne en santé publique : qui suis-je ? Rev Educ Med 1986;9:11-6.

[23] Cassou B. La formation en santé publique : un domaine en mutation. Actualité et dossier en santé publique 2006;55:15-6.

[24] Abric J-C. Pratiques sociales et représentations. 3th ed. Paris: Presses Universitaires de France; 2003.

[25] Vergés P. Approche du noyau central : propriétés quantitatives et structurales. In: Guimelli C, editor. Structures et transformations des représentations sociales. 1994. p. 233-53 [Vol. 277].

[26] Jodelet D. Représentation sociale : phénomène, concept et théorie. In: Moscovici S, editor. Psychologie sociale. Paris: Presses Universitaires de France; 1984.

[27] Vergès P. L'analyse des représentations sociales par questionnaires. Rev Fr Sociol 2001;42:53761.

[28] Rateau P, Rouquette ML. Introduction à l'étude des représentations sociales. $1^{\text {re }}$ éd. Presses Universitaires de Grenoble; 1998.

[29] Grimaud $O$, Charpak Y, Lombrail P. Recherche en santé publique : où est le pilote ? Rev Epidemiol Sante Publique 2014;62:177-8. 